\title{
El conflicto internacional en la zona gris: una propuesta teórica desde la perspectiva del realismo ofensivo
}

\author{
The international conflict in the gray zone: \\ A theoretical proposal from the offensive realism perspective
}

\author{
JAVIER JORDÁN \\ Universidad de Granada
}

\section{Como citar/Citation}

Jordán, J. (2018). El conflicto internacional en la zona gris: una propuesta teórica desde la perspectiva del realismo ofensivo. Revista Española de Ciencia Política, 48, 129-151. Doi: https://doi.org/10.21308/recp.48.05

\section{Resumen}

Este artículo ofrece una teorización del conflicto internacional en la zona gris, un término reciente en la literatura de estudios estratégicos. Para ello se delimita el concepto, identificando sus rasgos definitorios a partir de una revisión de la literatura existente. En segundo lugar, se enmarca el conflicto en la zona gris en la teoría del realismo ofensivo de John Mearsheimer, que estudia la política conflictiva entre grandes potencias y las estrategias utilizadas para incrementar el poder relativo. En tercer lugar, se identifican las líneas de acción estratégica desarrolladas en la zona gris, analizando sus pros y contras. Este tercer objetivo complementa y amplía la propuesta teórica de Mearsheimer, que explica el origen de la rivalidad entre grandes potencias, pero presta menos atención a cómo se desarrolla. De esta forma, el concepto de zona gris halla acomodo en la teoría realista de la política internacional y al mismo tiempo la enriquece.

Palabras clave: relaciones internacionales, estudios estratégicos, conflicto político, zona gris, realismo.

\begin{abstract}
This article offers a theorization of the international conflict in the gray zone, a recent term within the literature of strategic studies. To this end, the concept is settled by identifying its defining features based on a review of the existing literature. Secondly, the conflict in the gray zone is framed in John Mearsheimer's theory of Offensive Realism. It studies the conflictual politics between great powers politics and the strategies they use to increase their power relative to one another.
\end{abstract}


Thirdly, the strategic lines of action developed in the gray zone are identified, and their pros and cons are also analyzed. This third objective complements and extends Mearsheimer's theoretical proposal, which explains the origin of the rivalry between great powers but pays less attention to the development of this rivalry. Thus, the concept of gray zone finds accommodation within the realist theory of international politics and, at the same time, enriches it.

Keywords: international relations, strategic studies, political conflict, gray zone, realism.

\section{INTRODUCCIÓN}

En la comunidad de defensa norteamericana existe cierta indisciplina conceptual —criticada a menudo por ellos mismos— que lleva a la proliferación de buzzwords, términos de moda de utilidad analítica cuestionable y recorrido habitualmente efímero (Haas, 2017). Pero de vez en cuando aparece alguno digno de atención. Así sucede con el «conflicto en la zona gris», incluido por primera vez en la Quadrennial Defense Review norteamericana de 2010, y con el que se alude al espectro del conflicto político que separa la paz (blanco) de la guerra (negro).

La idea de fondo es simple y al mismo tiempo oportuna. Son cada vez más frecuentes las alusiones a un retorno a la Guerra Fría, cuando en realidad lo que se señalan son estrategias propias del conflicto en la zona gris, una de cuyas manifestaciones históricas fue precisamente el enfrentamiento bipolar (Green et al., 2017: 22). El conflicto en la zona gris es un fenómeno antiguo en la política internacional. Que reciba atención bajo ese término es un síntoma más de la creciente rivalidad entre grandes potencias.

La conceptualización del conflicto en la zona gris —al que en adelante nos referiremos simplemente como «zona gris»— es una tarea en curso. La literatura especializada —en su mayoría de carácter profesional (militar), no académica— aporta conceptualizaciones orientadas a la acción y no siempre bien definidas (Oldham, 2015; Votel et al., 2016). Hay excepciones como, por ejemplo, los trabajos de Mazarr (2015), Baqués (2017) o Wirtz (2017), pero todavía queda espacio para profundizar en su correcta delimitación teórica.

Este artículo se propone tres objetivos con el fin de que el término «zona gris» gane en potencia analítica. A tal fin, el trabajo se articula en cuatro apartados: en primer lugar, se delimita el concepto identificando sus rasgos definitorios a partir de una revisión de la literatura existente. En segundo lugar, se enmarca el concepto en una teoría superior en el ámbito de las relaciones internacionales. Para ello se recurre al realismo ofensivo de John Mearsheimer (2003), que estudia la política conflictiva entre grandes potencias y las estrategias utilizadas para incrementar el poder relativo. En tercer lugar, y siguiendo con la revisión de la literatura sobre zona gris, se identifican las líneas de acción estratégica desarrolladas en él, analizando sus pros y contras. Este tercer objetivo complementa y amplía la propuesta teórica de Mearsheimer, que explica el origen de la rivalidad entre grandes potencias, pero presta menos atención al modo como se 
desarrolla. De esta forma, el concepto de zona gris halla encaje en la teoría realista de la política internacional y al mismo tiempo la enriquece. Por último, se presentan brevemente los costes y riesgos que entrańa el conflicto en la zona gris, sugiriendo a partir de ellos futuras líneas de investigación.

\section{ELEMENTOS DEFINITORIOS DEL CONFLICTO EN LA ZONA GRIS}

La literatura sobre la zona gris es reciente, apenas tiene cinco años de antigüedad, y en muchos casos no ofrece una definición explícita del concepto, limitándose a señalar sus características principales. Por ello, antes de proponer una definición en este artículo, conviene identificar y sintetizar esos aspectos comunes.

1) Ambigüedad: ni relaciones pacíficas ni conflicto armado. En el conflicto en la zona gris la competición estratégica entre dos o más Estados (con sus respectivas diadas conflictivas) discurre por debajo del umbral de violencia política del conflicto armado menor (Baqués, 2017: 26). Según la definición del Uppsala Conflict Data Program (2018), un conflicto armado menor es aquel en el que se producen más de veinticinco muertes directas y menos de mil a lo largo de un año. De ello se deriva que una diada conflictiva conceptualizable como conflicto en la zona gris no puede serlo al mismo tiempo como conflicto armado. No a la vez, en la misma franja temporal, pero sí como una evolución de la disputa política a lo largo del tiempo.

En la zona gris el rol de la fuerza militar puede ir desde lo simbólico — celebración de grandes maniobras terrestres o vulneraciones del espacio aéreo que constituyan un show of force con finalidad intimidatoria - hasta acciones que bordean el conflicto armado abierto. Por ejemplo, el envío de «voluntarios» o de fuerzas militares con nacionalidad oculta en una situación prebélica o directamente de conflicto armado: desde los famosos «hombrecillos verdes» que ocuparon Crimea en febrero de 2014 a los pilotos rusos en la guerra de desgaste entre egipcios e israelíes en 1971 o en la de Corea en 1951 (Altman, 2016). Ese carácter fundamentalmente no violento del conflicto - salvo episodios puntuales y muy limitados - suele ser intencionado por parte de quienes lo libran y, en especial, de quien lo instiga (Brands, 2016). Se procura no cruzar líneas rojas que desemboquen en una contienda militar con costes altamente elevados y consecuencias imprevisibles (Mazarr, 2015: 58). Otro aspecto relevante de la zona gris es que al desarrollarse bajo el umbral bélico, un actor puede desafiar a otro más poderoso militarmente, siguiendo un cálculo acorde con la paradoja estabilidad/inestabilidad: que un Estado posea el dominio de la escalada en un nivel del conflicto no impide - $\mathrm{e}$ incluso invita a- que sus rivales lleven la pugna a estratos inferiores (Jervis, 1981: 31).

Utilizando como criterio cuantitativo el número de muertes en combate del Uppsala Conflict Data Program resultaría relativamente sencillo delimitar el 
conflicto en la zona gris del conflicto armado en un año concreto. La verdadera dificultad se encuentra en el otro extremo, en diferenciarlo de lo que se podría entender como decoro internacional, politics as usual, competencia bona fide, etc. En definitiva, de la política que discurre dentro de parámetros ampliamente aceptados. Nos topamos así con criterios inevitablemente subjetivos, y en ello radica precisamente una gran ventaja de esta opción estratégica: su ambigüedad. Una ambigüedad intencionada que dificulta tanto la identificación de las actividades hostiles propias de la zona gris como la articulación de estrategias de respuesta (Mazarr, 2015: 109-110).

2) Estrategias multidimensionales. En la literatura actual el conflicto en la zona gris es el espacio por excelencia del hybrid warfare, otro buzzword nacido en la comunidad de defensa norteamericana, aceptado en su variante de «amenaza híbrida" en declaraciones oficiales de la Alianza Atlántica y de la Unión Europea (Colom, 2018). El significado del hybrid warfare ha ido mutando — y abarcando realidades cada vez más diversas - a lo largo de los últimos ańos (Mansoor, 2012: 2; Baqués, 2015: 45-47; Jacobs y Lasconjarias, 2015: 259; Tenenbaum, 2015: 95-98). En algunas versiones actuales hace referencia a estrategias multidimensionales, integrales y de implementación sincronizada (Galeotti, 2016: 287; Cullen y Reichborn-Kjennerud, 2017: 9). De hecho, quizás sería preferible referirse a ellas de esa manera, dejando de lado el adjetivo híbrido y más aún el término warfare pues alude metafóricamente a algo que en realidad no es guerra (Biscop, 2015: 1; Charap, 2015: 52).

Pero yendo a lo esencial, en efecto un elemento definitorio del conflicto en la zona gris es ese empleo intencionado, multidimensional e integrado de diversos instrumentos de poder: políticos económicos, sociales, informacionales, diplomáticos y también militares (Adamsky, 2015: 37; Mazarr, 2015: 86; Freier, 2016: 4). La peculiaridad de lo militar en estas estrategias es su carácter fundamentalmente simbólico con intención coercitiva, utilizándose para señalar, intimidar y marcar territorio; y excepcionalmente para respaldar a terceros actores que sí recurren a la fuerza - y en ocasiones a gran escala — en el marco de una guerra por delegación (proxy war) en una diada de conflicto diferente a aquella en la que se está desarrollando el conflicto en la zona gris. Este es un aspecto que se analizará con más detalle en otro epígrafe. Lo característico por tanto del conflicto en la zona gris es el empleo mayoritario e integrado de herramientas no armadas.

3) Intereses sustanciales en juego. El conflicto gira en torno a intereses altamente valorados por quien se adentra en la zona gris. Los beneficios esperados superan los costes de abandonar la vía diplomática convencional, de cruzar eventuales líneas rojas o de testearlas. Dicha determinación constituye una ventaja si coincide con una "asimetría de intereses", gracias a la cual el Estado con menor cuota de poder relativo puede llegar a imponerse al asumir mayores sacrificios y riesgos que los que está dispuesto a soportar su rival por unos objetivos que no valora de la misma manera (Green et al., 2017: 30). El efecto de la asimetría 
de intereses también se aprecia fuera de la zona gris, en conflictos armados donde el débil vence al fuerte por razones similares (Mack, 1975: 179). Y, por último, la asimetría de intereses también afecta a la respuesta cohesionada o no de las alianzas, pues su elemento aglutinador es la percepción de amenaza, ligada a su vez a los intereses en juego (Walt, 1985: 35-37).

4) Gradualismo. Se trata de un conflicto a largo plazo donde abundan las acciones concebidas e interconectadas para lograr progresos de manera paulatina (Kapusta, 2015: 22). Ese gradualismo es una adaptación táctica que pretende evitar respuestas contundentes al tiempo que va modificando la situación estratégica por suma de efectos (Mazarr, 2015: 38; Echevarría, 2016: 11). El gradualismo refuerza la ambigüedad, pues la gravedad última y la interrelación de las distintas acciones no siempre resulta obvia a los decisores políticos del rival, a sus aliados y a sus respectivas opiniones públicas (Schadlow, 2014; Mazarr, 2015: 104; Brands, 2016).

A partir de estos cuatro elementos comunes en la literatura, se puede deducir la siguiente definición: la zona gris es un espacio intermedio en el espectro de conflicto político que separa la competición acorde con las pautas convencionales de hacer política, del enfrentamiento armado directo y continuado. El conflicto en la zona gris gira en torno a una incompatibilidad relevante para al menos uno de los actores. Las estrategias utilizadas son multidimensionales, de implementación gradual y con objetivos a largo plazo.

\section{EL CONFLICTO EN LA ZONA GRIS ENMARCADO EN EL REALISMO OFENSIVO}

La literatura sobre el conflicto en la zona gris apenas profundiza sobre sus causas estructurales. Se entienden por ellas los factores del sistema internacional que impulsan o desincentivan que la competencia entre Estados discurra en dicha franja del conflicto; no las causas específicas de un conflicto concreto en los niveles del individuo y del Estado — primera y segunda imagen de Kenneth Waltz (2001: 1-15), fundamentales en estudios de caso, pero con menos utilidad para la generalización de la teoría-.

Para estudiar ese origen en este artículo se ha optado por el realismo estructural, y en concreto por el realismo ofensivo de John Mearsheimer (2003). Llamativamente apenas se hace referencia a él en la literatura consultada sobre zona gris, lo cual es destacable porque, si bien el auge de la cooperación entre Estados y el mayor rol de las organizaciones internacionales en los años posteriores a la Guerra Fría invita a aplicar enfoques propios de la tradición liberal — como la paz democrática, la interdependencia compleja o el neoinstitucionalismo- el carácter crecientemente competitivo de la política internacional en nuestros días recuerda la validez de los principales postulados realistas.

Como es lógico, en política internacional no existe una "teoría del todo» que explique satisfactoriamente la enorme complejidad de las interacciones que se producen en 
ella, y por tanto se ha de recurrir a teorías concretas que ayudan a entender determinadas dimensiones de dicha complejidad. El realismo ofensivo es una de las teorías con mayor capacidad explicativa en lo que se refiere al conflicto entre grandes potencias (Toft, 2005: 400-403), y ello invita a introducir su perspectiva en la teorización de la zona gris. Esto no significa, sin embargo, que la propuesta teórica sobre la zona gris tenga poco que aportar a otras variantes del realismo, como por ejemplo el clásico o el neoclásico. Es compatible con ellos y, de hecho, resulta adecuado utilizarlos a la hora de analizar la acción exterior de un Estado concreto en un momento histórico determinado y su preferencia a la hora de elegir una u otra línea de acción estratégica en la zona gris. Esto es así porque el realismo ofensivo se encuadra en el realismo estructural de Kenneth Waltz (2001, 2010), que interpreta el comportamiento exterior de los Estados desde el nivel de análisis del sistema internacional. Se centra por tanto en las variables independientes, mientras que el neoclásico las asume y añade las variables intervinientes de los niveles de análisis del Estado y del individuo (siendo la variable dependiente el comportamiento exterior estatal).

Para los realistas estructurales, el sistema internacional se encuentra determinado por dos factores: 1) la anarquía internacional —ausencia de una autoridad supranacional que garantice de manera efectiva la seguridad de los Estados- y 2) la distribución de poder relativo entre los Estados. Según Waltz (2010: 127-128), los Estados permanecen atentos al equilibrio de poder para evitar la aparición de potencias hegemónicas. Cuando el equilibrio se rompe, los Estados con menos cuota de poder tienden a coaligarse para restaurarlo, por lo que una estrategia que persiga la hegemonía resulta a la larga contraproducente pues provocará el contrapeso del resto de actores. La estructura del sistema ofrece pocos incentivos para incrementar el poder sin medida. Este razonamiento de Waltz es compartido por otros autores realistas como Barry R. Posen (1984), Jack Snyder (1991), Charles L. Glaser (1994), Stephen Van Evera (1999), Shiping Tang (2010) y Stephen M. Walt (1985), aunque este último pone el acento en la percepción de amenaza antes que en la distribución de poder.

Mearsheimer cuestiona este planteamiento, al que califica de "realismo defensivo», y ofrece una explicación alternativa a la que da el nombre de "realismo ofensivo» (2003: 1-5; 2011: 424-427). Centra su análisis en las grandes potencias por ser estas las que ejercen mayor influencia sobre el sistema internacional. Para dicho autor, gran potencia es aquel Estado con suficiente poder militar para enfrentarse en una guerra abierta a la potencia más poderosa del mundo y, o bien vencerla o bien debilitarla seriamente. A día de hoy, la principal potencia del sistema es Estados Unidos; y a partir de ese patrón, se podrían entender como tales Rusia, China, India y, en menor medida, Reino Unido, Francia y Japón.

Tras identificar la unidad de análisis, Mearsheimer establece cinco principios (2003: 30-32):

1) El sistema internacional es anárquico; lo cual no es sinónimo de caos y desorden, sino ausencia de una autoridad centralizada por encima de los diferentes Estados. 
2) Por definición, las grandes potencias disponen de capacidades militares ofensivas con las que dañar gravemente a las demás.

3) Los Estados no conocen con seguridad las intenciones de los otros Estados. Quizás no sean hostiles, pero la incertidumbre resulta inevitable. A la vez, las intenciones benignas pueden tornarse inamistosas al cabo del tiempo.

4) La supervivencia constituye el objetivo básico de cualquier gran potencia, y más en concreto conservar su integridad territorial y su soberanía política.

5) Las grandes potencias se comportan como actores racionales, son conscientes de lo que ocurre en su entorno y calculan estratégicamente para sobrevivir en él.

Según Mearsheimer, la interrelación de estos cinco principios agudiza la competición y el recelo mutuo (ibid.: 32-34). Las grandes potencias pueden dañarse entre sí, no conocen con certeza las intenciones ajenas y no hay una autoridad externa de la que esperar protección. Ante una agresión, otros Estados ayudarán o no al atacado en función de sus respectivos intereses. El miedo los lleva a prestar atención a la distribución de poder en el sistema. Las grandes potencias solo se sienten seguras cuando son más poderosas que el resto, y como resulta difícil calcular el nivel «adecuado» de poder — no solo ahora sino en el medio y largo plazo-, cada una se siente impulsada a maximizar su cuota, a convertirse en abrumadoramente superior al resto de países o, dicho de otro modo, a erigirse en la potencia hegemónica de su región geográfica.

A su vez, las potencias que alcanzan la hegemonía procuran evitar la aparición de competidores dentro de su misma región, así como de potencias hegemónicas en otras regiones que les hagan sombra en el sistema internacional global o que puedan inmiscuirse en su área de influencia (Mearsheimer, 2014: 5-6). Para abortar el surgimiento de esos peer competitors las grandes potencias pueden actuar como «equilibradores de ultramar» (offshore balancers). Esa ha sido en parte -y debería ser aún más según Mearsheimer - la política seguida por Washington al fomentar el equilibrio contra Irán en Oriente Medio, contra Rusia en su antigua esfera de influencia en Europa del Este y Asia Central, y frente a China en Asia Pacífico (Mearsheimer y Walt, 2016: 82-83). Desde este punto de vista, podríamos sospechar que una Unión Europa cohesionada y con voz única en el plano exterior tampoco resultaría acorde con los intereses norteamericanos.

Para el realismo ofensivo la competición es permanente. Las potencias que han alcanzado la hegemonía regional procuran aumentar la distancia frente a potenciales competidores regionales y hacen lo posible para que no surjan pares en otras regiones del planeta. Es importante destacar este punto ya que la mayoría de la literatura norteamericana sobre el conflicto en la zona gris atribuye su ejercicio a potencias revisionistas — con nombre y apellidos: Rusia, China, Irán y Corea del Norte—, cuando lo cierto es que Estados Unidos también emplea estrategias propias de la zona gris (Robinson et al., 2018: 11-32). La acción exterior norteamericana resulta congruente con el realismo ofensivo ya que, según Mearsheimer (2003: 37), cuanto más poderoso sea un Estado más inclinado se sentirá a aprovechar su ventaja. 
Pasando a las estrategias, Mearsheimer señala que la guerra ha sido el principal medio de expansión territorial y de aumento de poder relativo a lo largo de la historia (ibid:: 168-233), aunque también reconoce que cada vez resulta más excepcional (ibid.: 147-152). No espera que una potencia en ascenso como China vaya a recurrir a la guerra de conquista contra sus vecinos en las próximas décadas (Brzezinski y Mearsheimer, 2005: 48). Coincide así, en líneas generales, con los autores realistas defensivos, para quienes la expansión territorial por la fuerza constituye una estrategia contraproducente, por la destrucción que provoca —y el enorme riesgo que entraña si los contendientes poseen armas nucleares- y por la resistencia que ofrecen las poblaciones a la ocupación (Snyder, 1991; Walt, 2011; Zielonka, 2012). A ello se podrían sumar los poderosos argumentos de la teoría liberal sobre paz democrática e interdependencia compleja (Rosecrance, 1986; Keohane y Nye, 1989; Maoz y Abdolali, 1989; Russet y Oneal, 2001). Sin embargo, Mearsheimer puntualiza que la guerra todavía puede ser considerada por algunos Estados una opción rentable a la hora de conquistar territorios de especial valor estratégico por los recursos que contienen o porque sirven de colchón defensivo (buffer zone). De modo que no conviene descartarla por completo a la hora de anticipar las estrategias estatales (2003: 150-151).

Pero lo habitual es que las potencias recurran a modos menos violentos. A veces frenan el ascenso de otras potencias — que tratan de incrementar el poder a su costa o de terceros- mediante estrategias de disuasión y de contención. En ese rol defensivo - pero explicable dentro del marco teórico del realismo ofensivo- los Estados pueden optar por el equilibrio de poder o por pasar la carga a otros (buck-passing). Con el primero, un Estado se suma a una coalición, mientras que en el segundo da un paso atrás para que otros asuman el contrapeso. El buck-passing es una estrategia eficiente porque desgasta al resto de potencias de manera indirecta — aumentando el poder relativo propio sin asumir costes-y porque permite secuenciar los esfuerzos en caso de que haya que hacer frente a varios rivales. No obstante, entrańa riesgos políticos por pérdida de influencia y por la posibilidad de que el contrapeso de los demás no logre frenar el ascenso de un rival (ibid.: 157-159).

Las grandes potencias también pueden recurrir al chantaje, tratando de obtener ganancias mediante la coerción sin emplear la fuerza a gran escala (ibid.: 138-139). En la literatura de estudios estratégicos es conocido como compellence (Schelling, 1966: 100). Puede funcionar en la interacción entre una gran potencia y un Estado menos poderoso, pero no tanto entre grandes potencias. Mearsheimer habla por último de una estrategia de desgaste del rival (bloodletting), generalmente en conflictos armados por delegación (proxy war). Es fácil recordar ejemplos de la Guerra Fría en Corea, Vietnam y Afganistán. Ambas estrategias — chantaje y desgaste- son perfectamente encuadrables en el conflicto en la zona gris aunque Mearsheimer no utilice explícitamente el término.

Se ha criticado a Mearsheimer por reducir demasiado el análisis al poder militar, descuidando otras herramientas de coerción, como por ejemplo las de carácter económico (Toft, 2005: 284). En efecto, su teoría se encuentra escasamente desarrollada en lo relativo a estrategias no bélicas, que en la actualidad son las más comunes. Por ello, 
la literatura reciente sobre zonas gris supone una aportación positiva a la teoría de Mearsheimer, ya que amplía conceptualmente el repertorio de estrategias utilizadas por las grandes potencias.

Y, viceversa, la propuesta de Mearsheimer explica desde el nivel de análisis del sistema internacional — con la capacidad de generalización que esto entrańa- por qué los Estados recurren al conflicto en la zona gris. Como se ha señalado al principio del epígrafe, se trata de un aspecto relevante no cubierto por la mayor parte de esta literatura, que tiende a ver esa competencia internacional como algo dado sin atender a su origen. La teorización del conflicto en la zona gris gana en profundidad explicativa desde el punto de vista causal al enmarcarse en el realismo ofensivo.

\section{LÍNEAS DE ACCIÓN ESTRATÉGICA EN LA ZONA GRIS}

Toda estrategia debe ajustarse a un equilibrio entre fines, modos y medios (Gray, 2013: 28). En el epígrafe anterior se ha explicado la raíz estructural de los fines —incrementar el poder relativo frente a Estados rivales_-, que lógicamente adquieren un contenido específico en cada caso concreto. Siguiendo con la revisión de la literatura, en este epígrafe se presta atención a los medios y, en particular, a los modos: a las líneas de acción estratégica empleadas en la zona gris que, para facilitar la exposición, se ordenan en apartados. No obstante, conviene recordar lo dicho en el primer epígrafe sobre su carácter integral y multidimensional, que parte de la literatura califica como «híbrido». Son líneas de acción complementarias y su empleo simultáneo multiplica los efectos sobre el rival.

1) Respaldo a la oposición politica del Gobierno adversario para generar confusión, agudizar fracturas y perturbar sus procesos de toma de decisiones. A día de hoy puede observarse en el respaldo mediático de Moscú a diversos partidos de extrema derecha anti-UE (Kramer y Speranza, 2017: 13-14). En la historia del siglo Xx abundan ejemplos más burdos de subversión política, y no solo durante el periodo de la Guerra Fría. Así, en 1901 Estados Unidos instigó una revuelta armada en los territorios colombianos del actual Panamá para favorecer su independencia y asegurar la construcción del Canal y el control de su área adyacente (Freier, 2016: 65).

En el nivel de menor intensidad de esta línea estratégica el apoyo se traduce en el mero respaldo político y mediático, y en el nivel más elevado en ayuda financiera o suministro de otro tipo de recursos necesarios para la actividad política opositora. Aquí se observa una vez más la ambigüedad. Objetivamente, en su nivel inferior, este tipo de actividades no tendrían por qué ser necesariamente hostiles, ni considerarse una injerencia en los asuntos internos de otro país. Pero en la zona gris cumplen un rol en combinación con otras líneas de acción estratégica. No es ningún secreto que la multiplicidad de actores y la complejidad de los problemas dificultan cada vez más la labor de gobierno democrático tanto a 
nivel estatal como supranacional, por ejemplo, en la Unión Europea o la Alianza Atlántica. Al mismo tiempo, la consabida urgencia del presente - con ciclos de información ininterrumpidos durante las veinticuatro horas del día-, unida al cortoplacismo marcado por el calendario electoral lleva a descuidar la planificación y el compromiso a largo plazo (ESPAS, 2015: 63). Estas circunstancias favorecen las acciones de zona gris destinadas a provocar disfunciones en los procesos de decisión política rivales. La ventaja es mayor cuando el actor que opta por el conflicto en la zona gris posee un proceso de toma de decisiones débilmente institucionalizado - sin controles y contrapesos propios de los sistemas democráticos- y no se siente constreñido por consideraciones éticas o legales (Kofman, 2017; Peco, 2017: 49).

2) Operaciones de influencia sobre la opinión pública internacional y sobre la opinión pública del adversario, construyendo y difundiendo metanarrativas —en lo posible de manera encubierta, a través de terceros- que afecten a los procesos políticos de otros Estados, favoreciendo los intereses de quien las promueve y deslegitimando las instituciones del bando rival. Es una línea de acción estratégica estrechamente vinculada a la anterior. Dichos metarelatos se difunden en el espacio público mediante informaciones sesgadas o falsas dirigidas a audiencias objetivo, favorables a esos puntos de vista. Este empeño puede verse amplificado en las redes sociales por la sinergia con otros individuos y grupos que compartan un adversario común o una causa semejante (Kragh y Åsberg, 2017: 779-800). En el caso de Rusia, la Comunidad de Inteligencia norteamericana ha acusado al canal de noticias Russia Today y a la Agencia de Investigación en Internet (Агентство интернет-исследований) de intentar influir en la última campaña electoral de Estados Unidos y en la de varios países de la Unión Europea (Office of the Director of National Intelligence, 2017: 4). Dicha agencia es calificada por otras fuentes como troll factory donde cientos de empleados escriben comentarios y noticias favorables a la política de Moscú desde perfiles online falsos (MacFarquha, 2018; Singer y Brooking, 2018: 111-114).

$\mathrm{Al}$ igual que en el punto anterior, las circunstancias del presente y las tendencias futuras ofrecen nuevas oportunidades en comparación con situaciones previas de zona gris, como la ya mencionada Guerra Fría, donde Estados Unidos y la Unión Soviética hicieron amplio uso de las operaciones de influencia (Snyder, 1997; Cull, 2008). La extensión de las redes sociales, combinada con adelantos en inteligencia artificial, incrementa sustancialmente el alcance de dichas operaciones. La difusión a través de internet de noticias manipuladas —en algunos casos replicadas decenas de miles de veces de manera automática por programas informáticos (bots) — llega de manera horizontal a amplias audiencias (Howard y Kollanyi, 2016: 1; Splidsboel-Hansen, 2017: 22). El empoderamiento de grupos e individuos, que gracias a la tecnología pueden coordinarse y actuar de manera efectiva y barata, también multiplica los efectos. Al igual que los avances tecnológicos, esta faceta del cambio social y político posee numerosos aspectos positivos, y a la vez crea oportunidades a las estrategias 
propias de la zona gris (National Intelligence Council, 2017: 25; Singer y Brooking, 2018: 181-216).

3) Coerción económica, mediante prácticas comerciales y financieras que refuercen la presión política. De nuevo se constata una gradación que va desde decisiones perfectamente legales y legítimas sobre la compra o venta de ciertos productos, a medidas de mayor calado como sanciones económicas o bloqueos (Mazarr, 2015: 59). Por ejemplo, en 2010 China suspendió temporalmente las ventas de minerales raros a Japón después de que los guardacostas nipones detuvieran a unos pescadores chinos en el marco de una disputa por aguas territoriales (Lai, 2018). Otro suceso similar, que refleja cómo la gradación puede iniciarse desde niveles muy sutiles de coerción, se produjo también en 2010 cuando China retrasó mediante inspecciones la compra de plátanos hasta que estos se pudrieron en los muelles filipinos con el fin de presionar a Manila en el contencioso sobre el Arrecife Scarborough, en el Mar del Sur (Green et al., 2018: 111). Más recientemente, el Gobierno chino ha utilizado las agencias de viajes de carácter estatal para redirigir su flujo turístico, penalizando a países como Corea del Sur (un descenso del $40 \%$ de turistas chinos) o a la República de Palao por motivos políticos (Coca, 2018).

4) Ciberataques contra entidades públicas y privadas, que además de amedrentar y generar confusión en el proceso de toma de decisiones políticas, airean la vulnerabilidad del adversario (Baqués, 2017: 22; Wirtz, 2017: 108). Pueden ser ataques de diversa consideración: desde denegaciones temporales de servicio en sitios web institucionales a acciones de mayor calado como los famosos ciberataques sufridos por Estonia en 2007 (Kramer y Speranza, 2017: 8-9). También pueden entrar en esta categoría - siempre que se efectúen en el marco de un conflicto en la zona gris - las acciones de ciberespionaje económico realizadas por las agencias de un Gobierno para abaratar los costes de investigación y desarrollo de su país, apropiándose de los avances obtenidos por las empresas de otros, una acusación que frecuentemente recae sobre las agencias de inteligencia chinas (Frier, 2016: 38). La dificultad de confirmar la autoría de este tipo de episodios se ajusta a unas estrategias que como ya se ha señalado se caracterizan por su ambigüedad (Rid y Buchanan, 2105: 4-5).

5) Acciones agresivas de inteligencia. Por extraño que suene, las actividades de inteligencia de unos Estados sobre otros forman parte de la política normal pues por estrechas que sean las relaciones suele haber terrenos de competencia política o económica donde la inteligencia proporciona ventaja competitiva (Lowenthal, 2012: 2-4). Sin embargo, en la zona gris esas actividades se tornan más agresivas: numerosos intentos de infiltración de los servicios rivales, campañas extensivas de ciberespionaje de entidades públicas y privadas del rival, acoso y expulsión de agentes, asesinato de defectores, etc. (Freier, 2016: 39). El envenenamiento con un arma química de un antiguo agente de los servicios de inteligencia rusos y de su hija en Reino Unido en marzo de 2018 sería un ejemplo de ese tipo de prácticas (Stewart, 2018). 
6) Disuasión militar coercitiva. Tradicionalmente en los estudios estratégicos coerción (compellence) y disuasión (deterrence) se han entendido como conceptos en cierto modo contrapuestos: amenaza o empleo limitado de la fuerza para que otro actor haga una cosa (coerción) o no haga otra (disuasión). Sin embargo, son complementarios pues dicha amenaza o empleo limitado de la fuerza puede incluir esa doble finalidad (Echevarría, 2016: 201-21). Aquí se aprecia una vez más la ambigüedad: lo que objetivamente es presentado como una medida puramente disuasoria y defensiva contiene además un sutil mensaje coercitivo. Por ejemplo, publicitando maniobras a gran escala cerca de la frontera de otro país, probando ostensiblemente nuevos sistemas de armas (misiles balísticos por parte de Irán), o violando repetidamente el espacio marítimo o aéreo de los vecinos, como por ejemplo hace Rusia en el Báltico (Adamsky, 2015: 38; Dalton, 2017: 313).

7) Hechos consumados, obteniendo una determinada ganancia en un solo paso y sin intención de retroceder. Alteran súbitamente el statu quo y colocan al adversario en una posición incómoda: ya no se trata de que las cosas sigan como siempre, sino de forzar un retorno a la situación previa. El fait accompli constituye además un fracaso puntual de la disuasión y un cuestionamiento de su validez en futuras ocasiones. La voluntad política y la capacidad militar que parecían firmes quedan en tela de juicio (Schelling, 1966: 44-45).

Para que el hecho consumado funcione la ganancia obtenida ha de ser limitada, de modo que la víctima prefiera dejarlo pasar a iniciar una escalada que puede acabar en guerra. Los hechos consumados son una estrategia común a la hora de ocupar territorios disputados entre dos o más Estados: entre 1918 y 2007 se produjeron 88 casos de ocupación militar unilateral de determinados territorios (63 de ellos después de 1945), 44 de los cuales dieron lugar a ganancias territoriales permanentes (Altman, 2018: 61; Altman, 2017: 881). La ocupación rusa de Crimea en 2014 es un caso de fait accompli exitoso; con un mínimo empleo de la fuerza quebró la estrategia disuasoria ucraniana y hasta de la propia OTAN (Wirtz, 2017: 108). Los hechos consumados no se limitan a la ocupación territorial. Incluyen otro tipo de actuaciones como, por ejemplo, los ataques aéreos de Israel contra las instalaciones nucleares de Irak en 1981 y Siria en 2007, que interrumpieron ambos programas de proliferación (Perlmutter et al., 2003; Makovsky, 2012).

8) Sliced salami tactics, concatenando acciones de bajo perfil que proporcionan ganancias graduales y que a la vez dificultan una reacción severa por parte del adversario (Mazarr, 2015: 34-36). En cierto modo, son una suma de pequeños hechos consumados. Su reducida entidad no justifica el empleo de la fuerza y deja margen al arreglo diplomático de las diferencias; mientras que si se ejecutasen de una vez abocarían a una crisis o incluso a una guerra. Son tácticas de erosión que además de generar ganancias degradan la credibilidad de la disuasión contraria. Con cada movimiento disminuye la probabilidad de que el adversario reaccione si no ha hecho nada hasta ese momento (Schelling, 1966: 
66-68). Un ejemplo sería la construcción de islas artificiales con instalaciones militares y la creación de zonas de identificación de defensa aérea por parte de Pekín en el mar del Sur de China tratando de afirmar su soberanía sobre esos espacios (Cooper y Shearer, 2017: 308; Holmes y Yoshihara, 2017: 323). Los hechos consumados y las tácticas salami son aún más atractivos si quien los protagoniza tiene el control de la escalada y cuenta con capacidades militares suficientes para imponerse en el nivel superior de conflicto. Siguiendo con el ejemplo anterior, el desarrollo de capacidades militares antiacceso y de denegación de área ( $\mathrm{A} 2 / \mathrm{AD}$ en sus iniciales anglosajonas) por parte de China en el mar del Sur incrementa las probabilidades de victoria en un conflicto limitado con la US Navy en la región y da más margen a la asertividad de China en la zona gris (Gompert et al., 2016: 3).

9) Guerras por delegación (proxy wars), donde se apoya militarmente a un Gobierno o a un actor armado no estatal en contra de un rival estratégico. La diada conflictiva tiene lugar en la zona gris porque no hay combates directos entre ambos Estados. Al igual que en las otras líneas de acción estratégica es posible una gradación de menos a más, que iría desde apoyar financieramente y prestar refugio a un tercero, a desplegar fuerzas militares propias como asesores no combatientes - $\mathrm{o}$ incluso combatiendo como «voluntarios» o con nacionalidad falsaen el propio teatro de operaciones (Altman, 2016). Las guerras actuales en Siria y Yemen son ejemplos claros de proxy war entre Irán y Arabia Saudí (Berti y Guzansky, 2014: 25-26). Durante la Guerra Fría hubo diversos casos de guerras por delegación como el apoyo norteamericano a los muyahidín afganos contra las fuerzas de ocupación soviéticas. Asimismo, Pakistán ha recurrido tradicionalmente al apoyo de grupos armados contra India en Cachemira en otro ejemplo claro de proxy war (Fair, 2014: 226-260).

\section{COSTES Y RIESGOS DE LAS ESTRATEGIAS EN LA ZONA GRIS}

El empleo coordinado, integral y sincronizado de las líneas de acción estratégica que se acaban de exponer proporciona ventaja frente un rival cuya estrategia defensiva se base fundamentalmente en la disuasión militar clásica, lineal y poco flexible frente al gradualismo y ambigüedad de las actividades en la zona gris. En el corto plazo las pérdidas no son de tal calado ni la amenaza se presenta con la suficiente nitidez y magnitud como para dar por fracasada la disuasión y abandonar la diplomacia (Wirtz, 2017: 107).

Pero adentrarse en la zona gris tampoco es una opción sin costes ni libre de riesgos. De hecho, sus líneas de acción pueden acabar generando reacciones por parte del resto de países que en último término debiliten la posición militar, política y económica de quien recurre a ellas (Mazarr, 2015: 71). En los casos más extremos las ganancias obtenidas mediante la ambigüedad y gradualismo pueden llevar a errores de cálculo sobre la seriedad de las líneas rojas del contrario y sobre la credibilidad de 
su disuasión (Freier, 2016: 27). Un ejemplo histórico fue la sorpresa de Hitler ante la declaración de guerra de Reino Unido y Francia tras la invasión de Polonia. Las acciones graduales del Tercer Reich en Austria, los Sudetes y Checoslovaquia alimentaron una peligrosa autocomplacencia en los mandatarios nazis que les llevó a malinterpretar las advertencias francesas y británicas en las últimas semanas de agosto de 1939 (Mazarr, 2015: 109-111).

Con el fin de sistematizar los riesgos de la zona gris, en la tabla 1 se comparan las posibles líneas de actuación con eventuales réplicas.

TABLA 1.

ACCIONES Y RESPUESTAS EN LA ZONA GRIS

Estrategias propias del conflicto en la zona gris

Posibles réplicas de los otros Estados

Contrainteligencia sobre actividades subversivas;

Respaldo a la oposición política del fomentar la transparencia de la financiación de

Gobierno rival para generar confusión, agudizar fracturas y perturbar sus procesos de toma de decisiones partidos y otros actores políticos: desenmascaramiento público del apoyo a grupos o partidos antisistema; apoyo a grupos de oposición del rival como represalia; denunciar públicamente la eventual corrupción y déficits democráticos del rival

Desenmascaramiento rápido y continuado de las noticias falsas dañando la imagen del Gobierno que

Operaciones de influencia sobre la opinión pública internacional y las promueve; descrédito de los medios de comunicación — sobre todo estatales_ que las sobre la opinión pública del adversario difunden; formación de las élites políticas y de la Administración frente a las operaciones de influencia; fomentar la conciencia social frente a la desinformación

Diversificación en otros mercados para evitar

Coerción económica dependencia estratégica; fortalecer la interdependencia económica con países aliados

Ciberataques contra entidades públicas y privadas Condena política pública; represalias legales; mejora de los sistemas de protección de TIC frente a nuevos ataques

Acciones agresivas de inteligencia Contrainteligencia; expulsión de diplomáticos Rearme para equilibrar las capacidades militares;

Demostraciones de fuerza con intención coercitiva formación y/o fortalecimiento de alianzas de contrapeso

Condena por parte de organizaciones internacionales:

Hecho consumado diplomacia coercitiva a través de sanciones económicas e incluso de empleo limitado de la fuerza para obligar el retorno al statu quo 


\begin{tabular}{ll}
$\begin{array}{l}\text { Estrategias propias del conflicto en la } \\
\text { zona gris }\end{array}$ & \multicolumn{1}{c}{ Posibles réplicas de los otros Estados } \\
\hline Tácticas salami & $\begin{array}{l}\text { No reconocimiento de las ganancias a través de } \\
\text { medidas diplomáticas o militares de carácter } \\
\text { simbólico; trazado de líneas rojas; fortalecimiento de } \\
\text { las alianzas ante conductas percibidas como agresivas }\end{array}$ \\
\hline Guerra por delegación & $\begin{array}{l}\text { Condena por parte de organizaciones internacionales; } \\
\text { sanciones económicas; ataques armados contra fuerzas } \\
\text { desplegadas sobre el terreno; pérdida de control de los } \\
\text { proxies }\end{array}$ \\
\hline
\end{tabular}

Fuente: elaboración propia.

Es importante destacar que las diversas estrategias de respuestas, que aparecen separadas en la tabla por razones de claridad expositiva, han de tener un carácter integral y sinérgico al aplicarse en la realidad, acorde con el conocido como comprehensive approach (Aaronson et al., 2011: 116-121). Sería objeto de otro artículo desarrollar cada una de las estrategias de réplica a las acciones en la zona gris. No obstante, conviene comentar, aunque sea brevemente, las posibles respuestas a las cuatro últimas por tratarse de las más reconocibles y las que mayor riesgo entrańan de que un conflicto en la zona gris escale a otro de naturaleza armada.

La demostración de fuerza coercitiva puede acabar incrementando el poder relativo del rival, incentivando su rearme o la mejora de su política de alianzas. Por ejemplo, las repetidas violaciones del espacio aéreo de los Países Bálticos por aviones militares rusos motivaron hace ańos el despliegue permanente de una fuerza de policía aérea de la OTAN que se mantiene en la actualidad, lo cual supone ciertamente un coste para los aliados, pero a la vez una presencia incómoda para el propio Kremlin. En casos extremos las demostraciones de fuerza coercitivas pueden provocar una escalada por parte del otro actor con riesgo asumido - conocida en la literatura de los estudios estratégicos como brinkmanship (Altman, 2018: 62)—. Por ejemplo, en noviembre de 2015 Turquía derribó un avión de combate ruso en la frontera siria tras denunciar varias violaciones de su espacio aéreo.

Más peligrosos resultan los hechos consumados (Van Evera, 1998: 10; Wirtz, 2017: 108). Como ya se ha señalado, el fait accompli intenta obtener una ganancia confiando en que la otra parte no reaccionará severamente, pero un error de cálculo puede precipitar los acontecimientos. Fue el fallo que cometió el régimen militar argentino cuando conquistó las islas Malvinas en abril de 1982 pensando que no habría respuesta armada, y dos meses después fue derrotado por los británicos (Altman, 2017: 68).

Por otro lado, la respuesta canónica al fait accompli es la diplomacia coercitiva, que consiste en el empleo de la amenaza y, llegado el caso, de un nivel limitado de fuerza con el propósito de que un actor interrumpa una línea de acción y/o vuelva a la situación previa a la alteración del statu quo (George, 1994: 8). Si el transgresor no cede y la 
otra parte aumenta el nivel de fuerza, la situación puede acabar en un conflicto armado en toda regla. Según Peter Viggo Jakobsen (2007: 235-244), de veintiún casos de diplomacia coercitiva entre Estados occidentales y diversos actores que traspasaron el statu $q u o$, cuatro acabaron en guerra y tres tuvieron éxito, pero con empleo limitado de la fuerza. El resto fracasó o cosecharon éxitos parciales con reincidencias posteriores; solo uno terminó con un retorno al statu quo sin requerir empleo alguno de violencia.

Cuando el objeto de la diplomacia coercitiva es una gran potencia el riesgo de guerra disminuye - por las graves consecuencias que entraña - pero aun así los costes pueden ser elevados, por ejemplo, en forma de sanciones económicas. El presidente Putin reconocía en 2016 que las sanciones estaban perjudicando seriamente al acceso a los mercados financieros internacionales y a muy grandes rasgos - hay otros factores como el bajo precio del petróleo- se estima que han reducido entre un 2\% y un 0,5\% el crecimiento del PIB ruso (Nelson, 2017: 8; Russell, 2018: 12).

Por otra parte, el trazado de líneas rojas precisas y reconocibles desincentiva los hechos consumados. Lo mismo se puede aplicar a las tácticas salami si dichas líneas abarcan aspectos parciales cuya defensa uno a uno no resulta cré́ble. En cuanto a estas últimas, se pueden contrarrestar mediante pequeñas acciones que, sin suponer una escalada del conflicto, transmitan un mensaje de no reconocimiento de los pequeños avances y reafirman el mantenimiento del statu quo. Por ejemplo, de vez en cuando buques de la US Navy navegan y realizan ejercicios a una incómoda distancia de los islotes artificiales chinos en el mar del Sur — en concreto, dentro de las doce millascon el fin de negar el estatus de territorio de soberanía que pretende concederles Pekín (Holmes y Yoshihara, 2017: 337).

Por todo ello, el aparente éxito de las tácticas salami —y más aún de los hechos consumados- puede activar y reforzar a la larga los mecanismos de disuasión del oponente, en conformidad con lo que Luttwak (2005: 7-9) denomina la lógica paradójica de la estrategia. Precisamente porque esas medidas funcionan el rival tratará de contrarrestar su efectividad. Y un modo de hacerlo consiste en mantener o recuperar el control de la escalada en el nivel superior a la zona gris mediante el desarrollo de capacidades militares que favorezcan la victoria en un conflicto armado limitado o incluso a gran escala.

Finalmente, la guerra por delegación es la línea de acción estratégica, si no más arriesgada - aunque también entrañe peligros—, sí la que mayor coste supone. Son varios los motivos: la elevada factura de un esfuerzo militar sostenido, el path dependency que obliga a seguir invirtiendo para justificar los esfuerzos realizados y evitar la imagen de derrota o debilidad, lo difícil de trabajar con aliados que persiguen su propia agenda y la posibilidad de que la potencia oponente responda con otra proxy war en otro escenario de conflicto (Dalton, 2017: 314).

\section{EPÍlOGO}

Poco después de que Rusia se anexionase Crimea, Walter Russell Mead (2014) escribía sobre "el retorno de la geopolítica" o, dicho de otro modo, la vuelta a una 
multipolaridad donde las grandes potencias se conducen de manera acorde con el realismo ofensivo. En cierta manera, las dos décadas posteriores al fin de la Guerra Fría constituyen una excepción histórica, con una gran potencia muy superior militarmente al resto. La creciente distribución de poder relativo — que muy probablemente se agudizará en los próximos años — supone una vuelta a la «normalidad» de la política internacional. Desde esta perspectiva no sorprende que grandes potencias como Rusia y China traten de afianzar sus respectivas esferas de influencia y quieran alterar el statu quo global construido por Estados Unidos.

Ese retorno a la multipolaridad no está exento de riesgos. En un libro reciente Graham T. Allison (2017: 154-184) advierte del peligro que conllevan las grandes transiciones de poder - la «trampa de Tucídides»- y plantea un escenario plausible de conflicto armado entre China y Estados Unidos en el horizonte de las dos próximas décadas. Allison no es determinista. Considera que ambas potencias pueden evitar la colisión si aplican las lecciones del pasado y aprenden de casos donde esas transiciones se resolvieron de manera pacífica.

A este respecto, Mearsheimer (2010: 389) no se hace ilusiones y asume que Pekín hará todo lo posible para reducir la influencia norteamericana en Asia Pacífico, aplicando su propia doctrina Monroe en la región. Al igual que Allison, Mearsheimer piensa que esa intensa competición entraña un importante riesgo de guerra (ibid.: 382). Sin embargo, la teoría sobre la zona gris expuesta en estas páginas ofrece una visión alternativa que en mi opinión — y sin descartar por completo lo posibilidad de una guerra limitada entre grandes potencias - resulta más congruente con la experiencia empírica de la década actual, que es la que nutre la mayor parte de la literatura revisada. No obstante, este sería un aspecto que profundizar en futuras investigaciones.

En este artículo se ha delimitado conceptualmente la zona gris y se han trazado las grandes líneas de los conflictos short of war entre grandes potencias; conflictos activos en la actualidad y que muy probablemente continuarán a medio y largo plazo. De este modo, la zona gris complementa de manera casi natural la teoría realista ofensiva, y es compatible con otras versiones del realismo como el clásico y el neoclásico, particularmente útiles a la hora de analizar casos concretos.

La contrastación empírica con la que Mearsheimer (2003: 168-233) respaldó en su momento la teoría del realismo ofensivo abarcaba la política entre grandes potencias de los últimos ciento cincuenta años, un periodo donde la guerra desempeñó un rol destacado. Sin embargo, el último capítulo del libro dedicado a las relaciones entre grandes potencias en el siglo XXI se centraba en los cambios futuros en la distribución de poder y las tensiones asociadas a ellos, mientras que las estrategias de las grandes potencias apenas recibían atención (ibid:: 360-402). Este artículo contribuye a rellenar ese vacío ofreciendo un marco teórico para analizar el modo como se materializa la rivalidad entre grandes potencias a día de hoy. Su empleo puede enriquecer líneas de investigación ya abiertas, como por ejemplo el estudio de las relaciones entre Rusia, Estados Unidos y los países europeos, la rivalidad creciente en la región de Asia Pacífico o la pugna por la primacía en Oriente Medio entre Arabia Saudí, Irán y Turquía. 
Por último, el artículo responde a una carencia de la literatura actual sobre zona gris: da por hecho la competición entre grandes potencias, pero no se detiene a explicar sus motivos últimos. Enmarcándolo en el realismo ofensivo, el conflicto en la zona gris adquiere una explicación estructural coherente que, aplicada a estudios de caso, es compatible además con la atención a las variables internas contempladas por el enfoque realista neoclásico.

\section{Referencias}

Aaronson, Michael, Sverre Diessen, Yves De Kermabon, Mary Beth Long y Michael Miklaucic. 2011. «NATO Countering the Hybrid Threat», PRISM, 2 (4): 111-124. Adamsky, Dmitry. 2015. Cross-domain coercion: the current Russian art of strategy. Institut Français des Relations Internationales. Proliferation Papers, 54. Disponible en: https://bit.ly/2aUq2UN [Consulta: 4 de mayo de 2018].

Allison, Graham. 2017. Destined for war. Can America and China escape Thucydides»s trap? Nueva York: Houghton Mifflin Harcourt.

Altman, Daniel W. 2016. «The long history of "green men» tactics and how they were defeated", War on the Rocks, March 17. Disponible en: https://bit.ly/2qtcjsr [consulta: 4 de mayo de 2018].

Altman Daniel W. 2017. «By fait accompli, not coercion: how states wrest territory from their adversaries», International Studies Quarterly, 61: 881-891. Disponible en: https://doi.org/10.1093/isq/sqx049.

Altman Daniel W. 2018. «Advancing without attacking: the strategic game around the use of force», Security Studies, 27 (1): 58-88. Disponible en: https://doi.org/1 $0.1080 / 09636412.2017 .1360074$.

Baqués, Josep. 2015. «El papel de Rusia en el conflicto de Ucrania: ¿̇la guerra híbrida de las grandes potencias", Revista de Estudios en Seguridad Internacional, 1 (1): 41-60. Disponible en: https://doi.org/10.18847/1.1.3.

Baqués, Josep. 2017. Hacia una definición del concepto «Gray Zone» (GZ), Documento de Investigación 2/2017. Instituto Español de Estudios Estratégicos.

Berti, Benedetta y Yoel Guzansky. 2014. «Saudi Arabia’s Foreign Policy on Iran and the Proxy War in Syria: Toward a New Chapter?», Israel Journal of Foreign Affairs, 8 (3): 25-34. Disponible en: https://doi.org/10.1080/23739770.2014.11446600.

Biscop, Sven. 2015. Hybrid Hysteria. Egmont Royal Institute for International Relations. Security Policy Brief, 64. Disponible en: https://bit.ly/2D2DFx4 [consulta: 4 de mayo de 2018].

Brands, Hal. 2016. "Paradoxes of the Gray Zone», Foreign Policy Research Institute, 5-2-2016. Disponible en: https://bit.ly/2AO7m3h [consulta: 4 de mayo de 2018].

Brzezinski, Zbigniew y John J. Mearsheimer. 2005. "Clash of the Titans», Foreign Policy, 146: 46-51.

Charap, Samuel. 2015. «The Ghost of Hybrid War», Survival, 57 (6): 51-58. Disponible en: https://doi.org/10.1080/00396338.2015.1116147. 
Coca, Nithin. 2018. «Chinese Tourists Are Beijing's Newest Economic Weapon», Foreign Policy, 26 September. Disponible en: https://bit.ly/2Ost4NZ [consulta: 20 de octubre de 2018].

Colom, Guillem. 2018. «Análisis de la actualidad internacional: contextualizando la guerra híbrida», Ciber Elcano, 32: 4-9.

Cooper, Zack y Andrew Shearer. 2017. "Thinking clearly about China»s layered Indo-Pacific strategy", Bulletin of the Atomic Scientists, 73 (5): 305-311. Disponible en: https://doi.org/10.1080/00963402.2017.1364005.

Cull, Nicholas J. 2008. The Cold War and the United States information agency: American propaganda and public diplomacy, 1945-1989. Nueva York: Cambridge University Press. Disponible en: https://doi.org/10.1017/CBO9780511817151.

Cullen, Patrick J. y Erik Reichborn-Kjennerud. 2017. Multinational capability development campaign project. Countering hybrid warfare project, understanding hybrid warfare. UK Ministry of Defence. Disponible en: https://bit.ly/2LcDWlC [consulta: 4 de mayo de 2018].

Dalton, Melissa G. 2017. «How Iran’s hybrid-war tactics help and hurt it», Bulletin of the Atomic Scientists, 73 (5): 312-315.

Echevarria, Antulio J. 2016. Operating in the gray zone: an alternative paradigm for U.S. military strategy. Carlisle: U. S. Army War College Press.

European Strategy and Policy Analysis System. 2015. Global trends to 2030: Can the EU meet the challenges ahead? Luxemburgo: Publications Office of the European Union.

Fair, C. Christine. 2014. Fighting to the end: The Pakistan army»s way of war. Oxford: Oxford University Press. Disponible en: https://doi.org/10.1093/acprof:oso/ 9780199892709.001.0001.

Freier, Nathan. 2016. Outplayed: regaining strategic initiative in the gray zone. Carlisle: U. S. Army War College Press.

Galeotti, Mark. 2016. «Hybrid, ambiguous, and non-linear? How new is Russia's 'new way of war'?», Small Wars and Insurgencies, 27 (2): 282-301. Disponible en: https://doi.org/10.1080/09592318.2015.1129170.

George, Alexander. 1994. "Coercive Diplomacy: Definition and Characteristics», en Alexander George y William E. Simons (eds.). The Limits of Coercive Diplomacy. Boulder CO: Westview Press.

Glaser, Charles L. 1994. «Realists as optimists: cooperation as self-help», International Security, 19 (3): 50-90. Disponible en: https://doi.org/10.2307/2539079.

Gompert, David C., Astrid Stuth Cevallos y Cristina L. Garafola. 2016. War with China. Thinking through the unthinkable. Santa Monica CA: RAND Corporation. Disponible en: https://doi.org/10.7249/RR1140.

Gray, Colin S. 2013. Perspectives on strategy. Oxford: Oxford University Press. Disponible en: https://doi.org/10.1093/acprof:oso/9780199674275.001.0001.

Green, Michael, Kathleen Hicks, Zack Cooper, John Schaus y Jake Douglas. 2017. Countering coercion in maritime asia. The theory and practice of gray zone deterrence. Washington D.C.: Center for Strategic and International Studies. 
Haass, Richard N. 2017. «13 International Relations Buzzwords That Need to Get Taken to the Woodshed», Foreign Policy, February 3. Disponible en web: https:// bit.ly/2koOnCx [consulta: 4 de mayo de 2018].

Holmes, James R. y Toshi Yoshihara. 2017. «Deterring China in the 'gray zone': lessons of the South China Sea for U.S. alliances", Orbis, 61 (3): 322-339. Disponible en: https://doi.org/10.1016/j.orbis.2017.05.002.

Howard, Philip N. y Bence Kollanyi. 2016. «Bots, \#strongerin, and \#Brexit: computational propaganda during the UK-EU referendum", Comprop Research Note 2016-1. Disponible en: https://bit.ly/2JGrWoV [consulta: 4 de mayo de 2018].

Jacobs Andreas y Guillaume Lasconjarias. 2015. «Hybrid warfare in the strategic spectrum: an historical assessment», en Guillaume Lasconjarias y Jeffrey A. Larsen (eds.), NATO"s response to hybrid threats. Roma: NATO Defense College.

Jakobsen, Peter V. 2007. "Coercive Diplomacy», en Alan Collins (ed.), Contemporary Security Studies. Oxford: Oxford University Press.

Jervis, Robert. 1981. The Illogic of American Nuclear Strategy. Ithaca, NY: Cornell University Press.

Kapusta, Philip. 2015. «The gray zone», Special Warfare, 28 (2): 19-25.

Keohane, Robert O. y Joseph S. Nye. 1989. Power and interdependence. Nueva York: Harper-Collins.

Kofman, Michael. 2017. «The Moscow school of hard knocks: key pillars of Russian strategy», War on the Rocks, 17-1-2017. Disponible en: https://bit.ly/2jwjvCj [consulta: 4 de mayo de 2018].

Kragh, Martin y Sebastian Åsberg. 2017. «Russia’s strategy for influence through public diplomacy and active measures: the Swedish case», Journal of Strategic Studies, 40 (6): 773-816. Disponible en: https://doi.org/10.1080/01402390.2016.1273830.

Kramer, Franklin D. y Lauren M. Speranza. 2017. Meeting the Russian hybrid challenge a comprehensive strategic framework. Washington, D. C.: Atlantic Council.

Lai, Christina. 2018. «Acting one way and talking another: china»s coercive economic diplomacy in East Asia and beyond», The Pacific Review, 31 (2): 169-187. Disponible en: https://doi.org/10.1080/09512748.2017.1357652.

Lowenthal, Mark M. 2012. Intelligence: from secrets to policy. Washington, D. C.: CQ Press.

Luttwak, Edward N. 2005. Para bellum: la estrategia de la paz y de la guerra. Madrid: Siglo XXI.

MacFarquhar, Neil. 2018. «Inside the Russian troll factory: zombies and a breakneck pace», The New York Times, 18-2 2018. Disponible en: https://nyti.ms/2HCy5ot [consulta: 1 de octubre de 2018].

Mack, Andrew. 1975. «Why big nations lose small wars: the politics of asymmetric conflict», World Politics, 27 (2): 175-200. Disponible en: https://doi.org/10.2307/ 2009880.

Makovsky, David. 2012. «The silent strike. How Israel bombed a Syrian nuclear installation and kept it secret», The Washington Institute, 17 de septiembre. Disponible en: https://bit.ly/2Pfrm7J [consulta: 1 de octubre de 2018]. 
Mansoor, Peter R. 2012. "Introduction: hybrid warfare in history», en Williamson Murray y Peter R. Mansoor (eds.), Hybrid warfare: fighting complex opponents from the ancient world to the present. Cambridge: Cambridge University Press. Disponible en: https://doi.org/10.1017/CBO9781139199254.001.

Maoz, Zeev y Nasrin Abdolali. 1989. «Regime types and international conflict, 18161976», Journal of Conflict Resolution, 33 (1): 3-35. Disponible en: https://doi.org/ 10.1177/0022002789033001001.

Mazarr, Michael J. 2015. Mastering the gray zone: understanding a changing era of conflict. Carlisle: U. S. Army War College Press.

Mead, Walter Russell. 2014. "The return of geopolitics: the revenge of the revisionist powers", Foreign Affairs, 93 (3): 69-79.

Mearsheimer, John J. 2003. The tragedy of great power politics. Nueva York: Norton.

Mearsheimer, John J. 2010. "The gathering storm: China»s challenge to US power in Asia», The Chinese Journal of International Politics, 3: 381-396. Disponible en: https://doi.org/10.1093/cjip/poq016.

Mearsheimer, John J. 2011. "Realists as idealists», Security Studies, 20 (3): 424-430. Disponible en: https://doi.org/10.1080/09636412.2011.599190.

Mearsheimer, John J. 2014. "Why the Ukraine crisis is the West»s fault», Foreign Affairs, 93 (5): 1-12.

Mearsheimer, John J. y Stephen M. Walt. 2016. "The case for offshore balancing. A superior U.S. grand strategy», Foreign Affairs, 95 (4): 70-83.

National Intelligence Council. 2017. Global Trends: Paradox of Progress. Washington D. C.: Office of the Director of National Intelligence.

Nelson Rebecca M. 2017. «U. S. Sanctions and Russia's Economy», Congressional Research Service, 17-2-2017. Disponible en: https://bit.ly/2D5sP9C [consulta: 4 de mayo de 2018].

Office of the Director of National Intelligence. 2017. «Background to assessing Russian activities and intentions in recent US elections: the analytic process and cyber incident attribution». Disponible en: https://bit.ly/2iRbS9b_[consulta: 4 de mayo de 2018].

Oldham, Chuck. 2015. «SOCOM: navigating the gray zone», Defense Media Network, 23-6-2015. Disponible en web: https://bit.ly/2yRNgUe_[consulta: 1 de octubre de 2018]

Peco, Miguel. 2017. «La persistencia de lo híbrido como expresión de vulnerabilidad: un análisis retrospectivo e implicaciones para la seguridad internacional», UNISCI Discussion Papers, 44: 39-54.

Perlmutter, Amos, Uri Bar-Joseph y Michael Handel. 2003. Two minutes over Baghdad. Londres: Frank Cass.

Posen, Barry R. 1984. The sources of military doctrine: France, Britain, and Germany between the World Wars. Ithaca, N.Y.: Cornell University Press.

Rid, Thomas y Ben Buchanan. 2015. «Attributing cyber attacks», Journal of Strategic Studies, 38 (1-2): 4-37. Disponible en: https://doi.org/10.1080/01402390.2014. 977382. 
Robinson, Linda, Todd C. Helmus, Raphael S. Cohen, Alireza Nader, Andrew Radin, Madeline Magnuson y Katya Migacheva. 2018. Modern political warfare. Current practices and possible responses. Santa Monica, CA: RAND Corporation. Disponible en: https://doi.org/10.7249/RR1772.

Rosecrance, Richard N. 1986. The rise of the trading state: commerce and conquest in the modern world. Nueva York: Basic Books.

Russell, Martin. 2018. "Sanctions over Ukraine. Impact on Russia», European Parliamentary Research Service Briefing, enero. Disponible en: https://bit.ly/2D0026h [consulta: 4 de mayo de 2018].

Russett, Bruce. M. y John Oneal. 2001. Triangulating peace: democracy, interdependence, and international organizations. Nueva York: Norton.

Schadlow, Nadia. 2014. «Peace and War: The Space Between», War on the Rocks, 18-82014. Disponible en: https://bit.ly/2mzXy2o [consulta: 4 de mayo de 2018].

Schelling. Thomas C. 1966. Arms and influence. New Haven, CT: Yale University Press.

Singer, P. W. y Emerson T. Brooking. 2008. LikeWar. The weaponization of social media. Nueva York: Houghton Mifflin Harcourt.

Snyder, Alvin A. 1997. Warriors of disinformation: American propaganda, soviet lies, and the winning of the cold war: an insider»s account. Nueva York: Arcade Publishing.

Snyder, Jack. 1991. Myths of empire. Domestic politics and international ambition. Nueva York: Cornell University.

Splidsboel-Hansen, Flemming. 2017. Russian hybrid warfare. A study of disinformation, Danish Institute for International Studies Report, 6. Disponible en: https:// bit.ly/2m3VQvl [consulta: 4 de mayo de 2018]

Stewart, Scott. 2018. "Russia sends a chilling message with its latest chemical attack», Stratfor, 13-3-2018. Disponible en: https://bit.ly/2P9gb0g [consulta: 1 de octubre de 2018].

Tang, Shiping. 2010. A theory of security strategy for our time: Defensive Realism. Nueva York: Palgrave Macmillan. Disponible en: https://doi.org/10.1057/ 9780230106048.

Tenenbaum, Élie. 2015. "Hybrid warfare in the strategic spectrum: an historical assessment», en Guillaume Lasconjarias y Jeffrey A. Larsen (eds.), NATO's Response to Hybrid Threats. Roma: NATO Defense College.

Toft, Peter. 2005. "John J. Mearsheimer: an offensive realist between geopolitics and power", Journal of International Relations and Development, 8: 381-408. Disponible en: https://doi.org/10.1057/palgrave.jird.1800065.

Uppsala Conflict Data Program. 2018. Definitions, Department of Peace and Conflict Research, Disponible en: https://bit.ly/2QmXy5Q [consulta: 4 de mayo de 2018].

Van Evera, Stephen.1998. "Offense, defense, and the causes of war», International Security, 22 (4): 5-43. Disponible en: https://doi.org/10.1162/isec.22.4.5.

Van Evera, Stephen. 1999. Causes of war: the structure of power and the roots of war. Ithaca, Nueva York.: Cornell University Press.

Votel, Joseph L., Charles T. Cleveland, Charles T Connett y Will Irwin. 2016. "Unconventional warfare in the gray zone», Joint Forces Quarterly, 80: 101-109. 
Walt, Stephen M. 1985. «Alliance formation and the balance of world power», International Security, 9 (4): 3-43.

Walt, Stephen M. 2011. "Nationalism rules», Foreign Policy, 15-7-2011. Disponible en: https://bit.ly/2ztz4Aq [consulta: 1 de octubre de 2018]. Disponible en: https:// doi.org/10.2307/2538540.

Waltz, Kenneth N. 2001. Man, the state and war. a theoretical analysis. Nueva York: Columbia University Press.

Waltz, Kenneth N. 2010. Theory of international politics. Long Grove, Illinois: Waveland Press Inc.

Wirtz, James J. 2017. «Life in the "gray zone": observations for contemporary strategists", Defense and Security Analysis, 33 (2), 106-114. Disponible en: https://doi. org/10.1080/14751798.2017.1310702.

Zielonka, Jan. 2012. «Empires and the modern international system», Geopolitics, 17 (3): 502-525. Disponible en: https://doi.org/10.1080/14650045.2011.595440.

Presentado para evaluación: 8 de mayo de 2018.

Aceptado para publicación: 25 de octubre de 2018.

\section{JAVIER JORDÁN \\ jjordan@ugr.es}

Profesor titular de Ciencia Política y director del Máster en Estudios Estratégicos y Seguridad Internacional de la Universidad de Granada. Es miembro del Grupo de Estudios en Seguridad Internacional (GESI). Ha sido investigador invitado en el Centro de Estudios Internacionales de la Universidad de Oxford (2001), en el Instituto Europeo de la London School of Economics (2002 y 2004), en el Instituto de Política Internacional del King's College of London (2003), así como en el Leonard Davis Institute for International Relations (2013) de la Universidad Hebrea de Jerusalén y en el Instituto Español de Estudios Estratégicos (2015-2016). Ha sido investigador principal del proyecto PIN 4-2017 «Sistema de análisis del entorno operativo del Ejército de Tierra», concedido por el Centro Mixto UGR-MADOC. Forma parte del equipo español en la Campaña de Desarrollo de Capacidades Multinacionales (MCDC) 2017-2018 «Countering Hybrid Warfare» (CHW2). 